\title{
6 Predicting shunt failure in children
}

\author{
Abhaya V. Kulkarni, MD, PhD \\ Division of Neurosurgery, The Hospital for Sick Children, Toronto, Ontario, Canada
}

$\mathrm{R}$ ossi et al. provide us with a retrospective review of risk factors for shunt revision at their center. ${ }^{1}$ After examining 18 factors, they found none to be statistically significant. The authors correctly remind us that the factors that truly influence shunt revision rate have not been definitively identified. They go further in their interpretation, however, to suggest, somewhat provocatively, that their results bring into question the validity of shunt revision rate as a quality metric. Specifically, they suggest that shunt revision rate does not meet the 3 criteria they believe are necessary for a quality metric: the metric 1) must make sense 2) be easily measurable; and 3) be modifiable by the surgeon. They base their argument on 1) the inability of their study to identify any factors associated with shunt revision; and 2) the assertion that previously proposed factors associated with shunt revision are nonmodifiable (i.e., beyond the surgeon's control) and have not been universally agreed upon. These points warrant closer examination.

First, there are several explanations for the authors' failure to find significant predictors (modifiable or nonmodifiable) of shunt revision. These include, for example, limited statistical power and a probable lack of sufficient heterogeneity in some of the variables due to this being a single-center study. Both of these limitations highlight the reasons why studies investigating quality metrics must involve multiple centers-not just to accrue sufficient sample size, but to accrue sufficient heterogeneity as well. Furthermore, it should be noted that Rossi et al. examined very few modifiable factors to begin with: only operative time, use of image guidance, and (arguably) intraoperative hemorrhage. This is just a tiny fraction of the potential modifiable factors available to study and was, no doubt, necessarily limited by the constraints of their database. So, ultimately their negative findings are not terribly robust.

Second, their claim that all proposed risk factors for shunt revision in the literature "are beyond the control of the surgeon" is debatable. Logically, this argument, taken to its extreme, requires us to believe that there is nothing that the surgeon does during surgery that influences shunt survival. This we know to be false prima facie, because the consequences of a carelessly placed proximal catheter or a preperitoneal distal catheter, or a shunt disconnection from a poorly tied knot are well known to us. These might be rare events, but they are generally preventable and speak directly to quality of care-and in fact these examples are all mentioned by the authors themselves. To definitively conclude that there are no modifiable factors for shunt revision would require a much larger, multicenter study in which all statistical variation in the shunt revision rate is fully explained by nonmodifiable factors, leaving no unexplained residual variation. Such a study has not been presented by Rossi et al. and, to my knowledge, does not exist.

The authors clearly acknowledge that modifiable factors for shunt revision do exist, because they state in their Discussion that they believe that a better quality metric would be the rate of "preventable shunt revisions." Although at first glance this seems a reasonable suggestion, I would argue that this fails the authors' second criterion of a good quality metric (i.e., it must be easily measurable).

Regardless of the above, however, Rossi et al. are correct to point out that the threshold for accepting quality metrics and risk adjustment must be very high. The consequences of such metrics are potentially quite serious in today's economic and legal environments. They demand a high degree of certainty and, importantly, community acceptance prior to such a metric's widespread use. As an example, the Society of Thoracic Surgery (STS) has developed a very robust process for risk adjustment for cardiothoracic procedures, and has gone even further, with voluntary public reporting of their results (http://www.sts. org/quality-research-patient-safety/sts-public-reportingonline/history-sts-national-database, last accessed June 8, 2015). Their database is truly national and now includes contributions from more than $90 \%$ of adult cardiac cen- 
ters in the US. The overall case volumes for hydrocephalus would make a similar effort much more difficult, but the STS model nevertheless provides a template that our community could at least attempt to emulate, and provides a roadmap for how this might be achieved.

http://thejns.org/doi/abs/10.3171/2015.6.PEDS15291

\section{Reference}

1. Rossi NB, Khan NR, Jones TL, Lepard J, McAbee JH, Klimo P Jr: Predicting shunt failure in children: should the global shunt revision rate be a quality measure? J Neurosurg Pediatr [epub ahead of print November 6, 2015. DOI: 10.3171/2015.5.PEDS15118]

\section{Disclosures}

The author reports no conflict of interest.

\section{Response}

\section{Paul Klimo Jr., MD, MPH, ${ }^{1-3}$ Nicholas B. Rossi, MD, ${ }^{1}$ Nickalus R. Khan, MD, ${ }^{1}$ and Tamekia L. Jones, $\mathrm{PhD}^{4}$}

${ }^{1}$ Department of Neurosurgery, University of Tennessee Health Science Center; ${ }^{2}$ Semmes-Murphey Neurologic \& Spine Institute; ${ }^{3}$ Le Bonheur Neuroscience Institute, Le Bonheur Children's Hospital; and ${ }^{4}$ Departments of Pediatrics and Preventive Medicine, University of Tennessee Health Science Center, Children's Foundation Research Institute, Memphis, Tennessee

There is nothing more deceptive than an obvious fact.

$$
\text { - Sir Arthur Conan Doyle }
$$

We thank Dr. Kulkarni for his comments, in which he recommends that multicenter studies are needed to accrue enough sample size and heterogeneity when investigating quality metrics. This might be true for finding overarching and general trends, but multicenter findings may not necessarily translate into applicability at an individual institution. For example, if a multicenter study showed that operative time was a predictor of shunt revision, but our own institution's data did not support this, how would this disparity be reconciled? Large databases indeed can detect small differences, but the magnitude of such difference needs to be scrutinized for clinical meaningfulness. If it requires hundreds or thousands of patients to detect a statistical difference, is such a finding useful in the dayto-day management of children with hydrocephalus at any one institution? Large multicenter studies assuming uniform surgical skills and uniform institutional patterns lose the element of "personalized" evidence relevant to specific patient populations. Whether multicenter or single-center data, each has its advantages and disadvantages.
Dr. Kulkarni states that we included "very few modifiable factors" and that the ones we used represent a "tiny fraction" of potentially modifiable factors. Yet he inexplicably provides no examples. Our list of variables was selected based on what others have previously investigated and what we thought were directly relevant to our clinical practice. One needs to be cautious about including an excessive number of additional variables because the risk of finding a statistical difference due to chance alone increases (i.e., a multiple-comparison problem).

We would naturally like to believe that we as surgeons command a significant, even dominant, control over the prevention of shunt failure. It is true that the current literature does not contain one or more universally accepted risk factors associated with revision, and those that are more commonly cited are nonmodifiable; namely age and etiology of hydrocephalus. But, as we state in our manuscript, we all know that there are some shunt failures that could have been prevented. At major US children's hospitals, shunt surgery is a relatively high-volume business, particularly in those institutions that support a residency and/or fellowship. Therefore, shunts are generally implanted and revised by well-trained and experienced neurosurgeons. Dr. Kulkarni actually bolsters our argument by pointing out that a preperitoneal catheter or an unsecured shunt are probably rare events in high-volume centers, and could certainly be part of what one would define as a "preventable shunt revision." A misplaced proximal or distal catheter and an improperly assembled and secured shunt can all be easily determined, prospectively or retrospectively, and thus measured.

So we return to his supposition that there exist many potentially modifiable factors in shunt surgery. But what are they? The literature to date has not shown a benefit to placement of the proximal catheter with image or ultrasound guidance, or to the choice of where to place the proximal catheter (i.e., frontal vs occipital). We simply do not know at this time what percentage of shunt revisions are preventable. Is it $10 \%$, or $50 \%$, or $75 \%$ ? Such data are institution and provider specific, and more work is needed before we contemplate using any revision data as part of a quality metric.

Contrary to Dr. Kulkarni's assertion, we admit that our study does not definitively conclude anything. We simply did not find any factor predictive of 90- or 180-day shunt revision rates at our institution over the last several years. Based on this negative study and the currently available literature, we maintain that the global shunt revision rate should not be used as a quality metric. As we go forward in this era of "outcomes," "quality," and "pay-for-performance," the creation and acceptance of quality metrics will be very challenging, probably more so than anticipated. The work and debate will continue. 